\title{
LA FORMACION DEL PERSONAL DE LAS ENTIDADES LOCALES Y REGIONALES EUROPEAS
}

35.088 .6

por

\author{
Alfonso Vizán Ferro \\ Miembro de la Mesa del Comité Directivo de Cuestiones Regionales \\ y Municipales del Consejo de Europa
}

SUMARIO: I. INTRODUCCION.-II. PANORAMA EUROPEO SEGUN LOS INFORMES DE LOS PAISES ENCUESTADOS: 1. AUStria. 2. Bélgica. 3. Dinamarca. 4. Finlandia. 5. Francia. 6. Grecia. 7. Irlanda. 8. Italia. 9. Luxemburgo. 10. Paf́ses Bajos. 11. Noruega. 12. Portugal. 13. Reino Unido. 14. República FeDERAL DE AlEMANIA. 15. SUECIA.-III. LA DIMENSION EUROPEA DE LA FORMACION PROFESIONAL.

\section{INTRODUCCION}

La Comisión de Estructuras y de Haciendas Locales de la Conferencia de Poderes Locales y Regionales de Europa (CPLRE) aprob6, en 29 de abril de 1982, un informe sobre «El Estatuto y la formación del personal de las Entidades locales y regionales», basado en los de los ponentes M. John Wint HER, Alcalde de Frederiksberg (Dinamarca), y Sir Duncan Lock, miembro del Consejo del Distrito de South Bucks (Reino Unido), y el cual ha dado lugar a la Resolución 131 (1982) de dicha Conferencia, adoptada en 20 de octubre último. Entre otros particulares, en esta Resolución se 
anima a las Entidades locales y regionales de las zonas fronterizas para que contribuyan a una más amplia formación europeísta de su personal, permitiendo a éste realizar prácticas en Entidades homólogas de allende las fronteras; y se encarga a la antedicha Comisión de Estructuras y Haciendas que organice en 1984 una Conferencia consagrada al estudio, a escala europea, de los problemas y de las aspiraciones de los empleados de las Entidades locales y regionales, con participación de representantes de los mismos. Además, se pide al Comité de Ministros del Consejo de Europa:

- que, como muestra de la cooperación entre los Países europeos más desarrollados y los menos avanzados, ponga en marcha, a partir de 1984, un programa de cooperación y de asistencia técnica para la formación del personal de dichas Entidades;

- que señale como objetivo de tal programa el ofrecer a ese personal la posibilidad de participar en cursos organizados por centros de formación de otros Países europeos, o en cursillos de prácticas (con intercambio, en su caso, de funcionarios) en Entidades similares de otros Estados miembros, con la finalidad de adquirir o de mejorar conocimientos en materia de administración o de finanzas locales y regionales, de servicios técnicos o de métodos modernos de gestión;

- que confíe a la CPLRE la tarea de coordinar este programa de cooperación técnica entre los Países interesados, y

- que conceda a este programa los medios necesarios (destinando, al menos, $250.000 \mathrm{FF}$ para subvencionar los estudios e intercambios antedichos) y encargue a la CPLRE la adjudicación de las bolsas a otorgar, conforme a las reglas que se preestablezcan y a reserva de una participación financiera suficiente por parte de las Entidades locales o regionales interesadas.

\section{PANORAMA EUROPEO SEGUN LOS INFORMES DE LOS PAISES ENCUESTADOS}

Las ponencias en cuestión se basan en las respuestas de sólo algunos de los Estados miembros del Consejo de Europa (más Finlandia) a sendos cuestionarios sobre el Estatuto y la formación del personal de que se trata; respuestas que, en cuanto a esta últi- 
ma (1) y siguiendo la a veces deficiente versión francesa del rapport de Sir Duncan LocK, ofrecen el panorama siguiente (2):

\section{AUSTRIA}

Respecto a la Administración regional, los Länder (o Estados de la Federación austríaca) se encargan de la formación de sus funcionarios (tanto de la básica como de la continua) a través de varios establecimientos de su creación. En cinco de los nueve Länder aquélla tiene lugar por medio de cursos, de seminarios y de enseñanzas impartidas en la Administración del propio Land; tres de ellos cuentan con una Universidad administrativa, y uno forma su personal en una Escuela ad hoc. Además, hay un Centro de prácticas para la formación continua.

En cuanto a la formación del personal de las Corporaciones locales, en seis Länder corre a cargo de Escuelas o Academias de Administración, con ayuda especialmente de las asociaciones de Entidades locales. Paralelamente, esa formación está también asegurada por los antedichos establecimientos de los Länder o por medio de seminarios y cursos organizados por el sindicato de funcionarios locales o por los partidos políticos. Estos últimos son particularmente activos en el campo de la formación continua.

El tipo de formación varía según la esfera de actividad y el nivel de conocimientos que se pretende impartir respecto a ella. La formación básica se refiere principalmente al Derecho constitucional y administrativo austríaco, al régimen del personal y al de las funciones que hayan de asumirse. La formación continua (dispensada mediante cursos, seminarios y cursillos monográficos) tiene por objeto no sólo la actualización de los conocimientos exigidos para el desempeño de la función, sino el aprendizaje de nuevas técnicas de gestión y de relaciones públicas.

(1) En cuanto a la primera de dichas ponencias (es decir, la relativa al Estatuto del personal) puede verse un resumen de la misma en la revista CUNAL (número de diciembre de 1982).

(2) Téngase en cuenta que las respuestas corresponden generalmente a los años 1980 y 1981, por lo que algunos de sus datos pueden haberse modificado con poste. rioridad. Se omiten las referidas a España, por su falta de interés para los lectores. 


\section{BÉLGICA}

Para la formación administrativa, las Provincias organizan cursos en nueve ciudades, cuyo objeto es la preparación y el perfeccionamiento de los funcionarios del sector público, y especialmente de la Administración municipal. Tales cursos duran dos años, a cuyo término se obtiene un diploma. El Instituto provincial de Amberes expide, además, tras un ciclo de cinco años, el diploma de licenciado en Derecho administrativo.

Para la formación de la Policía municipal existen también Centros provinciales, donde se siguen cursos teórico-prácticos que duran un año. Tras ellos puede pasarse a los cursos para mandos, que duran dos años.

Por otra parte, desde 1977 existe el Instituto de Asuntos Públicos, con sede en Charleroi, patrocinado por la Universidad de Mons $\mathrm{y}$ otros establecimientos de enseñanza superior, y subvencionado principalmente por el Ayuntamiento de aquella primera ciudad. En su seno hay una sección municipal, donde se siguen cursos que completan los organizados por las Provincias: el de formación administrativa superior dura dos años; ocho meses, el destinado a los agentes de la Policía municipal, y cuatro, el especial para asistentes sociales. Paralelamente, el Instituto organiza seminarios y jornadas de estudio para los funcionarios de los diferentes servicios, y desarrolla programas de investigación por encargo de las Comunidades regionales.

\section{Dinamarca}

En 1940, entre varias Entidades locales se creó el «Danks kommunalkursus", como Centro de formación del personal municipal, encargado de la producción del material pedagógico y de organizar las pruebas finales de los estudios correspondientes. Años después, todos los Municipios de cierta importancia se habían afiliado a dicho Centro, el cual se rige por un Consejo de nueve miembros (cuatro, nombrados por las delegaciones locales de aquél; cuatro, por las asociaciones de Municipios, y uno, por la federación de empleados municipales). La formación está descentralizada, pues dicho Centro cuenta con treinta sucursales, a las que acuden los cursillistas una vez por semana, en jornada de tarde.

Superados con éxito los estudios del primer año, puede acce- 
derse a la formación de carácter permanente que imparte la Escuela Nacional de Administración Pública, creada en 1962 con el fin de preparar al personal administrativo del sector público (central o local), desde auxiliares hasta directivos con título universitario. Se rige por un Consejo de dirección de dieciséis miembros (siete, representantes del Estado; seis, de las asociaciones de Entidades locales, y tres, de las asociaciones de funcionarios), al que asesora un Comité consultivo en el que participan representantes de la federación de empleados antedicha. El presupuesto de la Escuela se nutre con subvenciones del Gobierno y de las Entidades locales, y con el importe de las tasas académicas.

Las enseñanzas corren a cargo de funcionarios del Estado y de las Corporaciones locales con formación universitaria, a los que se conceden permisos al efecto y que, por término medio, dedican dos horas por semana a su labor docente.

Por otro lado, desde 1967 existe una Escuela Superior Municipal, patrocinada por las asociaciones de Entidades locales, pero con personalidad jurídica y edificios propios, capaces de acoger 120 alumnos a la vez. Su finalidad es organizar cursillos breves, de tres a cinco días de duración y cuarenta participantes como máximo, entre los que también se admite a concejales. Las materias tratadas cubren todos los campos de actividad de las Corporaciones locales, pero se atiende fundamentalmente a los aspectos administrativos de la misma. Las enseñanzas corren también a cargo de funcionarios de la Administración central o local, en colaboración con especialistas en las materias a tratar y con las secretarías de las referidas asociaciones, que participan en la organización de los diferentes cursillos y publican Memorias periódicas sobre ellos. Unas 8.000 personas asisten anualmente a los mismos, de las que un tercio suelen ser concejales.

\section{Finlandia}

La «Asociación Finlandesa de Entidades Locales» ha creado, en 1956, el «Instituto de Formación Municipal», cuyas enseñanzas corren a cargo de expertos de dicha Asociación y de concejales. En 1979 quedaron abiertos 355 cursos o cursillos, a los que asistieron 18.515 personas.

Los gastos de este Instituto se cubren en su mayor parte por los derechos de inscripción en los cursillos, ya que las subvenciones 
del Gobierno y los demás ingresos sólo representan el 10 por 100 de aquellos gastos.

\section{FRANCIA}

Un cambio decisivo, en cuanto a la formación del personal de las Entidades locales, ha tenido lugar en 1972, con la creación, por Ley, del "Centre de Formation des Personnels Communaux» (CFPC), cuya finalidad fundamental es la que indica su nombre, junto con el perfeccionamiento profesional de todas las categorías de empleados municipales y la organización de pruebas, concursos y oposiciones relativos a los mismos, así como mejorar la calidad de los servicios: que prestan las Entidades locales.

El CFPC tiene el carácter de establecimiento público intermunicipal y está bajo la tutela del Ministerio del Interior. Se rige por un Consejo de Administración presidido por un Alcalde y compuesto de 25 miembros, de los que diez representan a los Municipios, diez a los empleados municipales, dos al Ministerio citado y uno al de Educación, más dos expertos independientes. Sus cursos ordinarios son gratuitos, y sus gastos de funcionamiento se cubren mediante una contribución obligatoria de los Ayuntamientos y de sus servicios municipales autónomos, aparte de subvenciones de otras fuentes cuando se trata de cursos especiales. En cambio, el Estado no satisface las que debieran correr a su cargo según la Ley, y últimamente ha surgido un conflicto entre el Consejo de Administración y el Ministerio del Interior, por negarse éste a autorizar una subida del 0,08 por 100 en el tipo de cotización de las Entidades locales, que venían aportando a razón del 0,92 por 100 de sus gastos de personal.

Este Centro tiene su sede en París, pero funciona de forma descentralizada, mediante una red de sucursales regionales, cada una de ellas especializadas en preparaciones determinadas, con la consiguiente organización de cursos y elaboración del material de estudio. A estos fines cuentan con la asistencia de comités consultivos regionales y provinciales, en los que participan los miembros de las Corporaciones interesadas. Las enseñanzas son impartidas por profesores dependientes del Estado y por funcionarios locales especialmente calificados para el caso.

El CFPC ha tenido a su cargo la formación de 56.000 personas. en 1980 , y cuenta con unos 800 empleados permanentes. 


\section{GRECIA}

Las referencias a este País son muy parcas. Se señala únicamente que «los empleados de las Entidades locales, una vez nombrados, pueden seguir cursos de enseñanza general y asistir a seminarios de perfeccionamiento, con arreglo a programas de formación preparados y puestos en práctica por el Gobierno».

\section{IRLANDA}

Las Entidades locales de importancia destinan uno de sus funcionarios, con dedicación plena, a organizar la formación o el perfeccionamiento del personal de todas las categorías (incluidos los trabajadores manuales), mediante cursos, conferencias, prácticas de laboratorio o de taller, etc. Por otra parte, existe el «Institute of Public Administration", organismo privado que se dedica, entre otras tareas, a la formación de personal, especialmente el de las Entidades locales, y que, además de los cursos ordinarios al efecto, organiza eventualmente otros de tipo coyuntural. Otros dos organismos, "The Industrial Training Authority» (para aprendizajes técnicos) y «The National Institute for Physical Planning and Research», organizan también cursos para los empleados municipales.

Estos tres Institutos están subvencionados por el Estado (el primero, con 700.000 libras para 1982, aunque sus ganancias se calculan en el doble); pero la Administración central sólo interviene en el funcionamiento de los mismos a través de sus representantes en el Comité de formación del personal de las Corporaciones locales («Local Authorities Training Comittee», en el cual están también representados los sindicatos y dichas Corporaciones), a cuyo juicio el Instituto de Administración Pública, antes mencionado, somete su programa de actuación para el año siguiente.

\section{ITALIA}

No existen aquí, a nivel nacional, instituciones para la formación profesional de los funcionarios de las Entidades locales, si bien el Ministerio del Interior organiza cursos destinados a los secretarios de dichas Entidades, a fin de actualizar sus conocimientos en materia legal y administrativa. Por su parte, varias Regiones se dispo- 
nen a organizar cursos de formación para el personal de sus respectivos órganos de gobierno (Consejo y Junta regional).

\section{LUXEMBURGo}

Tampoco existe aquí una institución central encargada de la formación de que se trata, la cual corre a cargo de los propios Municipios interesados, bajo la tutela del Ministerio del Interior.

\section{Países Bajos}

Como instituciones de formación profesional se citan las Escuelas de Administración Local ("Stichting Samenwerkende Bestuursscholen») de ámbito provincial, en una de las cuales, la de la Provincia de Drenthe, se desarrollan cursos para los miembros de las Corporaciones locales, y el Instituto de Ciencias Administrativas ("Institut voor Bestuur Wetenschappen»), creado en 1939 por la Unión de Municipios Neerlandeses. Pero en 1977, las citadas Escuelas y el Departamento de formación y enseñanza del mencionado Instituto se fusionaron al efecto de crear el CIVOB (Instituto Central de Formación de la Administración Pública), con sede en La Haya, y dirigido por un Consejo en el que están representados, además de la referida Unión y las autoridades locales, los sindicatos de funcionarios y los Ministerios del Interior y de Educación. Este Instituto, al que también corresponde la organización de las pruebas para la selección y nombramiento del personal de que se trata, se sostiene mediante aportaciones de las Entidades locales, subvenciones del Estado y tasas académicas. Un Reglamento modelo, negociado entre dichas Entidades y el colectivo nacional de los funcionarios a su servicio, sirve de pauta a la mayoría de las mismas en orden al régimen de licencias y de ayudas al personal que asiste a cursos de formación o de perfeccionamiento en interés del servicio.

Por su parte, la Unión de Municipios Neerlandeses cuenta con unas 60 personas encargadas de la organización de seminarios y cursos destinados a funcionarios y miembros de las Corporaciones locales. 


\section{NORUEGA}

Fundamentalmente, la formación y el perfeccionamiento están asegurados aquí por el KOU («Fondo Municipal para la Formación y el Desarrollo"), creado y financiado por las propias organizaciones de funcionarios y por la Asociación Noruega de Corporaciones Locales, que tiene a su cargo la secretaría del Fondo. Este organismo prepara cursos y stages descentralizados (con la colaboración de dichas Corporaciones), que, en su mayor parte, se desarrollan por correspondencia y por medio de seminarios de corta duración.

Por otra parte, en el «Instituto Municipal» de Oslo y en algunos Institutos provinciales (Distriktshogskoler) pueden seguirse cursos más largos sobre materias de Administración local.

\section{Portugal}

Recientemente se ha creado en Coimbra un Centro de Formación del Personal de las Entidades locales, donde podrán seguirse cursos permanentes en régimen de internado. Se proyecta un cuadro docente matriz constituido por unos treinta especialistas, a los que se trata de convertir en «formadores de formadores». Se prevén asimismo cursillos descentralizados de corta duración.

\section{REINO UNIDO}

Según la circular del Consejo Nacional Paritario (febrero de 1978), la formación profesional de los funcionarios de que se trata debe estar abierta a todas las categorías de los mismos, y las Entidades locales están obligadas a facilitarles aquélla mediante bibliotecas apropiadas y la asistencia a cursos ad hoc.

Dichas Entidades seleccionan a los empleados idóneos para seguir tales cursos, satisfaciéndoles el sueldo, los derechos de inscripción y los gastos suplementarios; pero reservándose el derecho de exigir su reintegro si aquéllos no se presentan a los exámenes o si dejan el servicio de la Entidad antes de cierto plazo.

Aparte de los cursos organizados por las propias Corporaciones locales, éstas financian al "Local Government Training Board", organismo creado en 1968 para asegurar una formación de nivel y amplitud adecuados a la demanda, por parte de aquéllas, de personal 
calificado de las distintas categorías. Algunos de sus cursos, con ayuda de los establecimientos locales de enseñanza, pueden seguirse fuera de las horas de trabajo de los participantes. Este organismo expide certificados y diplomas correspondientes a las enseñanzas: que imparte.

Debe añadirse que, para determinadas materias, los cursos pueden seguirse por correspondencia.

\section{República Federal de Alemania}

Como en Austria, el sistema alemán de formación del personal de las Entidades locales y regionales es heterogéneo, pues varía de. un Land a otro. En general, se distinguen tres niveles de formación: la escolar o académica, la profesional técnica (interna, para el personal administrativo, y externa, para servicios especiales) y el perfeccionamiento de la misma en consonancia con la evolución legal y las nuevas tecnologías.

Para la mencionada «interna», la mayor parte de los Länder disponen de su propio Instituto. La denominada "externa" se recibe en cursos organizados por las Universidades $u$ otros centros de enseñanza general.

En cuanto a los cursos de perfeccionamiento, las Entidades locales suelen conceder licencia a sus empleados que deseen seguirlos, y toman a su cargo los gastos correspondientes; pero, en la mayoría de los Länder, los beneficiarios están obligados a reintegrar tales gastos si dejan el servicio de la Entidad en los dos años siguientes.

Como rasgo general se señala también que en la República Federal Alemana las perspectivas de promoción en la carrera están ligadas a la eficacia profesional, más bien que a los diplomas o a los. cursos de perfeccionamiento seguidos. Estos se consideran generalmente como meras ayudas al funcionario para mantener o mejorar tal eficacia.

\section{SUECIA}

En cuanto al personal de las Entidades municipales, la Asociación de Municipios Suecos ha creado, hace treinta años, un Instituto para la formación y perfeccionamiento de dicho personal, situado en Sitguna, pequeña localidad vetusta, a 35 kilómetros de Estocol- 
mo; y cuenta, además, con un equipo especializado en la preparación de material de estudio y en la organización de cursillos descentralizados.

Análogamente, respecto al personal de los «Consejos de Condado" (especie de Diputaciones provinciales), la Federación Sueca de los mismos ha fundado una Escuela próxima a la capital, y cuenta también con un equipo dedicado a las cuestiones de formación, el cual prepara los programas y el material de estudio (manuales, filmes, etc.) de los cursos y seminarios que organiza para los empleados y para los miembros electivos de los Consejos (alrededor de 3.000 participantes al año).

El Instituto y la Escuela antedichos disponen de instalaciones modernas y de alojamientos para cien participantes. Sus recursos se nutren exclusivamente con las subvenciones de la Asociación y Federación citadas, y con los derechos de inscripción en los cursos, que se satisfacen por las Entidades de que proceden los alumnos que asisten a los mismos.

Aparte de la citada Escuela, todos los Consejos de Condado tienen su propio Centro de formación y perfeccionamiento para empleados y miembros corporativos; si bien algunos de aquéllos se han unido para la elaboración de programas comunes a fin de unificar tal formación a nivel provincial.

Según datos de un informe oficial relativo al año 1975, alrededor del 27 por 100 de los empleados de las Entidades locales (Municipios y Consejos de Condado) siguen algún curso o cursillo cada año; y la carga que ello supone para las mismas se estima en el 0,5 por 100 de sus gastos de personal.

Por si fuera poco, los sindicatos de funcionarios organizan también cursos para la formación del personal de que se trata.

\section{LA DIMENSION EUROPEA DE LA FORMACION PROFESIONAL}

En el capítulo final del rapport de Sir Duncan Lock -cuyas conclusiones se recogen en las ya transcritas peticiones finales de la Resolución de la CPLRE - se plantea la cuestión de si existe o no la necesidad de una formación, a escala europea, del personal de las Entidades locales. En pro de la afirmativa cabe argüir, además de la creciente importancia de los aspectos internacionales de las fun- 
ciones de aquéllas, la conveniencia de cierto grado de formación común para facilitar la cooperación intraeuropea y para que los: funcionarios de países con un sistema de Administración local menos avanzado puedan aprovecharse de las experiencias de otros más adelantados en este campo.

Para lograr tal formación común podría pensarse en la necesidad de un Centro internacional ad hoc; pero la creación del mismo tropezaría con graves dificultades financieras y de medios personales; por lo que, en las actuales circunstancias, parece más aconsejable el sistema de conferencias, seminarios y cursos internacionales sobre materias específicas, como los que vienen organizándose ya. por diversas instituciones de ese carácter (por ejemplo, la Conferencia Tecnológica Permanente de las Entidades Locales Europeas, que se ha ocupado recientemente de problemas tales como la recogida de basuras, el ahorro de energía, las compras agrupadas y el transporte de materiales tóxicos). Además, convendría elaborar un programa de cooperación técnica entre los países europeos de distinto nivel de desarrollo en materia de formación de personal; programa que debería financiarse, en lo posible, por el Consejo de Europa y que se asemejaría al aplicado por el mismo en beneficio del Gobierno portugués tras la revolución de 1974, con la finalidad de posibilitar al personal de las Entidades locales y regionales la asistencia a cursos de formación seguidos en Centros extranjeros.

A juicio del autor del informe, la puesta en práctica de tal programa de cooperación técnica entre países de distinto nivel de desarrollo respondería a la prioridad otorgada a ésta por el Comité de Ministros del Consejo de Europa en su sesión de octubre de 1980, y se inscribiría en el marco del Dictamen número 21 de la CPLRE. (sobre el Segundo Plan a Medio Término de dicho Consejo) y de su Resolución 113 sobre "Los progresos de la integración europea». 


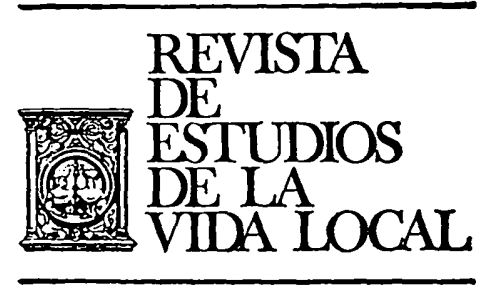

III. ESTADISTICA 
REVL-1983, núm. 218. VIZAN FERRO, ALFONSO. LA FORMACION DEL PERSONAL DE LAS ENTI...

REVL-1983, núm. 218. VIZAN FERRO, ALFONSO. LA FORMACION DEL PERSONAL DE LAS ENTI... 